\title{
Artifact Removal from EEG with a Preprocessing for Preserving Desired Bands
}

\author{
Ching-Tai Chiang \\ Department of Computer and Communication \\ National Pingtung Institute of Commerce \\ Pingtung, Taiwan \\ cctai@npic.edu.tw \\ Chen-Sen Ouyang \\ Department of Information Engineering \\ I -Shou University \\ Kaohsiung, Taiwan
}

\author{
Rong-Ching Wu \\ Department of Electrical Engineering \\ I -Shou University \\ Kaohsiung, Taiwan \\ line 4: e-mail: name@xyz.com \\ Lung-Chang Lin, Rei-Cheng Yang \\ Department of Pediatrics \\ Kaohsiung Medical University Hospital \\ Kaohsiung, Taiwan
}

\begin{abstract}
Artifacts such as eye movements, muscle, electrocardiogram (EKG) and line noise may cause severe contamination in Electroencephalogram (EEG). The independent component analysis (ICA) is the general method to remove the artifacts within electroencephalographic traces. Desired bands in specific EEG analysis, however, may be seriously eliminated in the deflation procedure. A preprocessing with extraction of desired bands in the ICA is presented to prevent the desired bands from cancellation in the process of artifact rejection. Thus, the desired bands in EEG can be completely preserved while removing the artifacts. Some real EEG recordings are provided to demonstrate the effectiveness of the proposed method.
\end{abstract}

Keywords-Electroencephalogram (EEG), Artifact, Independent component analysis

\section{INTRODUCTION}

Severe contamination within Electroencephalogram (EEG) recordings by eye movements, muscle, electrocardiogram (EKG) and line noise is a serious problem for EEG analysis. The recorded EEG data are often preprocessed to remove serious and obvious motion artifacts through visual inspection. However, when the recorded EEG data is limited, or muscle and line noise occur too frequently, as in some patient groups, discarding the contaminated EEG segments results in an amount of information loss and may be impracticable for clinical diagnosis.

Independent component analysis (ICA) has successfully been used in biomedical systems [1]. Several studies dealing with ICA-based biomedical systems have been reported during the last decades [2]. The approach for measuring statistical independence between random variables is different for various methods. One group of algorithms such as SOBI [3], COM1 and JADE [4] use cumulant-based techniques. Alternately, some algorithms, such as InfoMax, FastICA or PICA [5] measure independence via mutual information. The ICA-based algorithms extract the independent components from the recorded EEG data to perform blind separation of source signals. Different artifacts that are hidden in the contaminated EEG data may be isolated to different ICA components. After discarding the components, we can reconstruct the corrected EEG data with artifact removal from the remaining ICA components in the deflation procedure. However, the artifact-related components often contain desired bands for specific EEG analysis, such as alpha rhythms. The alpha rhythms are an important phenomenon of the brain. Alpha waves are naturally produced by the brain when we are in a relaxed state, and hence, the alpha activity is an index of relaxation, but alertness. Unfortunately, in order to remove the artifacts, the alpha rhythms may be seriously eliminated in the deflation procedure.

In this paper, a method for preserving desired bands in artifact removal from EEG is presented. A preprocessing with extraction of desired bands in the ICA can prevent the desired bands from cancellation in discarding the artifactrelated ICA components. The observed EEG data are provided to demonstrate the efficacy of the proposed technique for the artifact rejection from EEG.

\section{EXTRACTION OF DESIRED BANDS AS A PREPROCESSING IN ICA}

A preprocessing with extraction of desired bands is presented for artifact removal using ICA. We observe records of electrode signals

$$
\mathbf{x}(k)=\left[x_{1}(k), \ldots, x_{m}(k)\right]^{T}
$$

where $\mathrm{k}$ is a discrete time, $\mathrm{m}$ denotes the number of electrodes on scalp, and $(\bullet)^{T}$ means transpose of the vector. From an unknown MIMO (multiple-input/multiple-output) mixing system, we consider the observed signals $\mathbf{x}(k)$ are linearly mixed with $\mathrm{n}$ brain sources by an unknown matrix $\mathrm{A}$, that is,

$\mathbf{x}(k)=\mathbf{A s}(k)+\mathbf{v}(k)$

where the brain source vector

$\mathbf{s}(k)=\left[s_{1}(k), \ldots, s_{n}(k)\right]^{T}$ 
and $\mathbf{v}(k)$ is a unknown vector representing errors or noise. In general, the alpha rhythms are an important phenomenon of the brain, and can be viewed as a desired band in the EEG analysis. However, the important signals, such as alpha rhythms, may be seriously eliminated during artifact removal based on ICA.

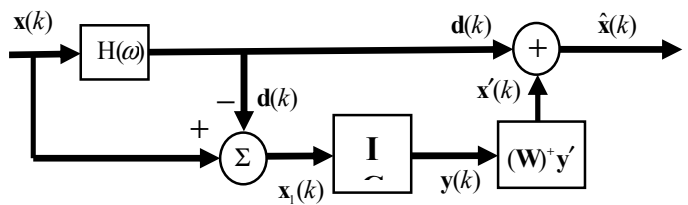

Figure 1. ICA for artifact removal with a preprocessing of band extraction

In order to prevent the desired bands from elimination during artifact removal, a preprocessing of extracting desired bands is inserted in a standard ICA, as shown in Fig. 1. The preprocessing consists of a bandpass filter with a transfer function $\mathrm{H}(\omega)$. Through the bandpass filter, the desired signals $\mathbf{d}(k)$ can be obtained via digital signal processing technique. The preprocessed data without desired signals may be represented as

$$
\mathbf{x}_{1}(k)=\mathbf{x}(k)-\mathbf{d}(k)
$$

Then, the blind source separation (BSS) in the ICA can be executed by a separating matrix $\mathrm{W}$, and the independent components $\mathbf{y}(k)$ can be obtained as

$$
\mathbf{y}(k)=\mathbf{W} \mathbf{x}_{1}(k)
$$

Finally, corrected EEG signals can then be derived as

$$
\mathbf{x}^{\prime}(k)=(\mathbf{W})^{+} \mathbf{y}^{\prime}(k)
$$

where the superscript + denotes the Moore-Penrose pseudoinverse, and $\mathbf{y}^{\prime}$ is the matrix of independent waveforms, ${ }^{\mathbf{y}}$, with rows representing artifact sources set to zero. Adding the desired signals, we obtain the totally corrected EEG signals is

$$
\hat{\mathbf{x}}(k)=\mathbf{x}^{\prime}(k)+\mathbf{d}(k)
$$

It is obvious that the desired signals are preserved completely in the EEG signals with artifact rejection.

\section{EXPERIMENTS}

The EEG data were provided by Kaohsiung Medical University Hospital, Kaohsiung medical University. EEG recordings were collected from 21 scalp electrodes, all referred to the left mastoid. The Amuse algorithm [6] is employed to implement the ICA.

A. Ocular artifacts

Fig. 2 shows a 4-sec portion of the contaminated EEG time series (left), its ICA component activations (middle), and the corrected EEG signals (right) obtained by removing the artifacts from the contaminated EEG data. The ocular artifact in the EEG data (left) is isolated to ICA component 1 (middle). The scalp topography, shown in Fig. 3(a), indicates that the component 1 account for the spread of ocular artifact to frontal sites (Fp1, Fpz, Fp2). After removing the artifact from the frontal channels, the corrected EEG signals reveal the alpha waveform near $10 \mathrm{~Hz}$ that was masked by the ocular artifact.

\section{B. Muscle artifacts}

The muscle artifacts occurred between $0-2 \mathrm{sec}$ in the temporal sites T5 and T6 in Fig. 2 (left). This artifacts are isolated to ICA components 15 and 16 (middle). The scalp map in Fig. 3(b) confirms the muscle artifacts are mostly concentrated in the T5 and T6 electrodes. Removing them from the raw data, we can observe that the corrected signals (right) at the T5 and T6 channels reveal underlying EEG activity obscured by the muscle artifacts in the raw data (left). C. EKG artifacts

The cardiac contamination appeared at the waveform of $\mathrm{P} 3$ electrode in the original EEG data (left). This artifact is isolated to ICA component 2. In Fig. 3 (c), the scalp map of component 2 captures the EKG artifacts to P3 position. The underlying EEG activity obscured by the cardiac artifacts in the raw data can be observed in the corrected signal trace at the P3 channel in Fig. 2 (right).

\section{Line noise artifacts}

There are two types of line noise contamination in Fig. 2 (left). The first type is the EEG signals at F3 and P4 channels. The artifact is isolated to ICA components 18 and 19 (middle). The scalp maps in Fig. 3(d) and Fig. 3(e) confirm this type of line noise is concentrated at F3 and P4 electrodes. The EEG signal at $\mathrm{Pz}$ channel shows the second type of line noise, and the ICA component 14 reveals this artifact. The scalp map of the component in Fig. 3(f) clearly captures this type of line noise to Pz position.

E. Spectra comparison

Fig. 4 plots the spectra comparison between the proposed method and the standard ICA algorithm (Amuse [6]) for artifact removal. Figs. 3(a), (b) and (c) clearly show that the alpha rhythms (8-13 Hz) at FP1, FPz and FP2 channels, respectively, are seriously cancelled for the removal of ocular artifacts, while the proposed method preserves the alpha rhythms completely.

\section{SUMMARIES}

This paper presents a preprocessing with extraction of desired bands for ICA-based artifact removal in EEG data. The proposed method can effectively remove the artifacts and preserve the desired bands completely. Some real EEG recordings are provided to illustrate the efficacy of the proposed method for the artifact removal.

\section{REFERENCES}

[1] Hyvarinen A., Kahunen J., and Oja E., 2001, Independent Component Analysis, John Wiley \& Sons, Ltd, UK.

[2] Sanei S., and Chambers J.A., 2007, EEG Signal processing, John Wiely \& Sons, UK.

[3] Belouchrani A., Abed-Meraim K., Cardoso J. F., and Moulines E., 1997, "A blind source separation technique using second order statistics”, IEEE Trans. Signal Process., 45(2), pp.434-444.

[4] Cardoso J.F., and Souloumiac A., 1996, "Jacobi angles for simultaneous diagonalization", SIAM Journal Matrix Analysis and Applications 17, pp. 161-164. 
[5] Karvanen J., and Koivunen V., 2002, "Blind separation methods based on Pearson system and its extensions", Signal Processing, Elsevier 82, pp. 663-673.
[6] Tong L., Inouye Y., and Liu R., 1993, "Waveform-preserving blind estimation of multiple independent sources," IEEE Transaction on Signal Processing, 41(7), pp. 2461-2470.
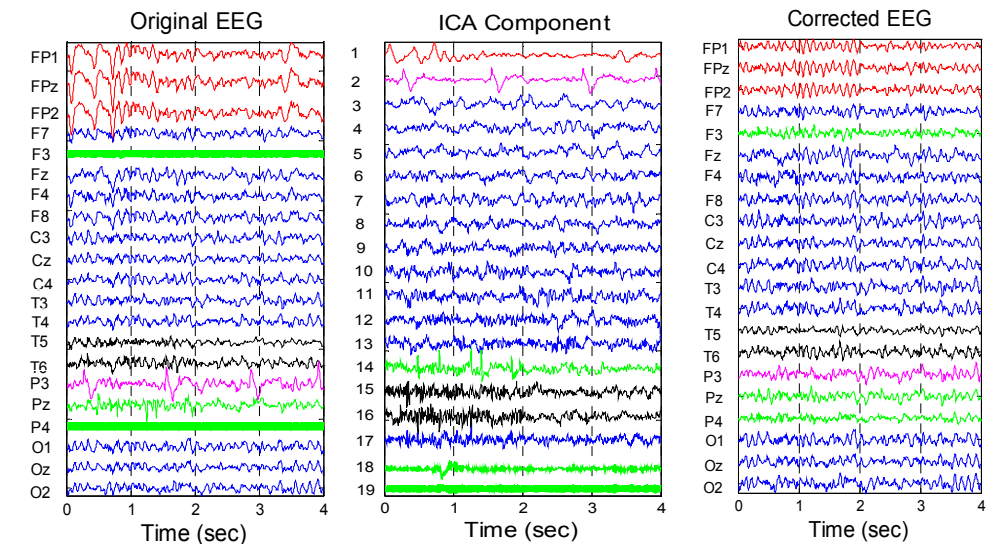

Figure 2. (left) A 4-sec portion of an EEG time series. (middle) ICA components accounting for ocular artifacts, cardiac signals, muscle noise and line noise sources. (right) The EEG signals corrected for artifacts by removing the seven selcted components (ICA 1, 2, 14, 15, 16, 18 and 19)

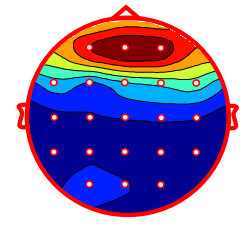

(a) ICA 1

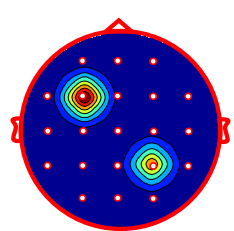

Figure 3. Scalp topography of artifact-related ICA components.

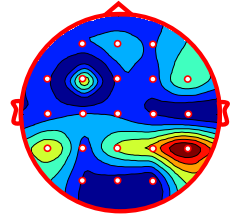

(b) ICA 15,16

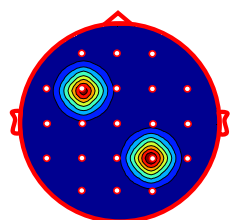

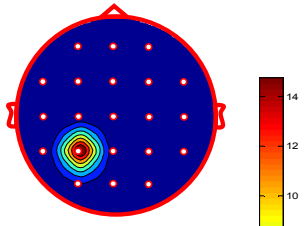

(c) ICA 2

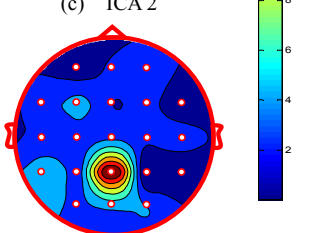

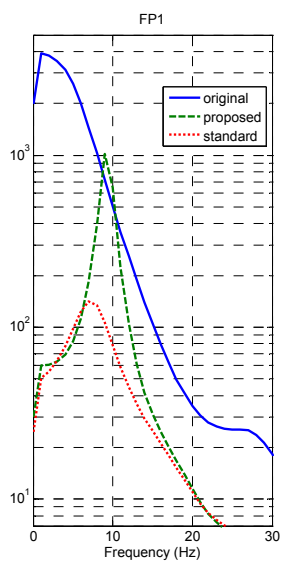
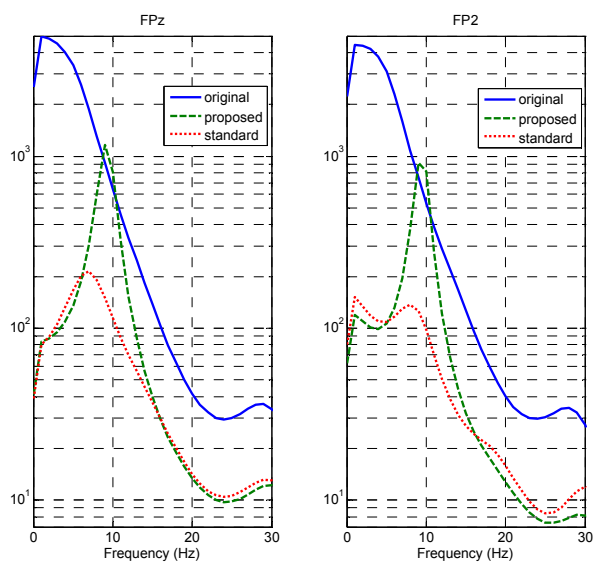

Figure 4. EEG spectra comparison at three channels. The proposed method preserves the alpha rhythms, while the standard method eliminates the alpha rhythms seriously. 